\title{
MS26-02 | The Power of Analogy in Physics: From Faraday Waves through Soft Matter to Complex Metallic Alloys
}

Lifshitz, Ron (Raymond and Beverly Sackler School of Physics \& Astronomy, Tel Aviv, ISR)

As early as 1985, Landau free-energy models [1-3] and density-functional mean-field theories [4] were introduced in an attempt to explain the stability of quasicrystals, with only partial success if any. It is only in recent years, that great progress has been made in under-standing the thermodynamic stability of quasicrystals in such simple isotropic classical field-theories. Much of this has happened thanks to insight from the experimental observation of quasicrystalline order in diverse systems ranging from fluid dynamics to soft condensed matter. The key to unlocking the stability puzzle was in the realization that more than a single length scale was required, but more importantly in figuring out how to introduce these multiple scales into the models, and identifying the remaining requirements $[5,6]$. We and others have since managed to produce Landau and other mean-field theories with a wide range of quasicrystals as their minimum free-energy states, and have also confirmed some of these theories using molecular dynamics simulations with appropriately designed interpar-ticle potentials [7-14]. I shall give a quick overview of the quasicrystals that can be stabilized in these theories-in systems of one or two types of particles, in two and in three dimensions-and attempt to identify a trend that might be emerging in going from Landau theories to more realistic density-functional mean-field theories. It remains an open question whether this trend may eventually lead to understanding the stability of quasicrystals in complex metallic alloys.

This research is supported by Grant No. 1667/16 from the Israel Science Foundation.

[1] P. Bak, Phys. Rev. Lett. 54, 1517 (1985).

[2] N.D. Mermin, S.M. Troian, Phys. Rev. Lett. 54, 1524 [Erratum on p. 2170] (1985).

[3] P.A. Kalugin, A.Yu. Kitaev, L.C. Levitov, JETP Lett. 41, 145 (1985).

[4] S. Sachdev, D.R. Nelson, Phys. Rev. B 32, 4592 (1985).

[5] R. Lifshitz, H. Diamant. Phil. Mag. 87, 3021 (2007).

[6] R. Lifshitz, D. Petrich, Phys. Rev. Lett. 79, 1261 (1997).

[7] K. Barkan, H. Diamant, R. Lifshitz, Phys. Rev. B 83, 172201 (2011).

[8] A.J. Archer, A.M. Rucklidge, E. Knobloch, Phys. Rev. Lett. 111, 165501 (2013).

[9] K. Barkan, M. Engel, R. Lifshitz, Phys. Rev. Lett. 113, 098304 (2014).

[10] C. V. Achim, M. Schmiedeberg, and H. Löwen, Phys. Rev. Lett. 112, 255501 (2014).

[11] P. Subramanian, A.J. Archer, E. Knobloch, A.M. Rucklidge, Phys. Rev. Lett. 117, 075501 (2016).

[12] S. Savitz, M. Babadi, R. Lifshitz, IUCrJ 5, 247 (2018).

[13] M.C. Walters, P. Subramanian, A.J. Archer, R. Evans, Phys. Rev. E 98, 012606 (2018).

[14] S. Savitz, R. Lifshitz, "Self-assembly of body-centered icosahedral cluster crystals", in preparation (2019). 